\title{
COVID-19, función suprarrenal y corticosteroides
}

\author{
COVID-19, adrenal function, and corticosteroids
}

\section{Pinzón-Tovar $A^{1}$, Castellanos-Bueno $R^{2}$}

${ }^{1}$ Médico Internista, Universidad Surcolombiana. Endocrinólogo, Universidad Militar Nueva Granada de Colombia. Coordinador, Comité de Hipófisis, Asociación Colombiana de Endocrinología, Diabetes y Metabolismo. Presidente, Capitulo Alto Magdalena, Asociación Colombiana de Medicina Interna. Docente, Medicina Interna, Universidad Surcolombiana. Endocrinólogo, Hospital Universitario Hernando Moncaleano Perdomo Neiva. Dirección Científica, Endho, Colombia.

${ }^{2}$ Médico Internista y Endocrinólogo. Comité de Hipófisis, Asociación Colombiana de Endocrinología, Diabetes y Metabolismo. Profesor

Asociado, Departamento de Medicina Interna, Universidad Industrial de Santander.

Autor de correspondencia: Alejandro Pinzón Tovar Correo electrónico: alepyto@yahoo.com

\section{Resumen}

La pandemia por SARS-nCoV-2 elevó muchos interrogantes sobre su efecto en múltiples sistemas. Los datos han sido tomados de infecciones previas por coronavirus, estudios epidemiológicos observacionales y explicaciones que demuestran plausibilidad biológica. El compromiso que produce el virus SARS-nCoV-2 no escapa al campo de la endocrinología y en este documento se consigna la relación que existe entre la función suprarrenal, su respuesta a los procesos infecciosos y el uso de corticosteroides en la COVID-19.

Palabras clave: COVID-19, pandemia, hormonas de la corteza suprarrenal.

\begin{abstract}
SARS-nCoV-2 pandemic raised many questions about its effect on multiples systems. Data has been taken from previous Coronavirus infections, observational epidemiological studies and explanations that demonstrate biological plausibility. The commitment that the SARS-nCoV-2 virus produces does not escape the field of endocrinology and in this document the relationship that exists between adrenal function, its response to infectious processes and the use of corticosteroids in COVID-19 is recorded.
\end{abstract}

Keywords: COVID-19, Pandemic, Adrenal cortex hormones.

\section{COVID-19}

El nuevo coronavirus 2 (SARS-nCoV-2) produce la infección viral conocida como COVID-19. Esta patología afecta las vías respiratorias, compromete la membrana alveolocapilar y desarrolla un síndrome de dificultad respiratoria aguda (SDRA); en situaciones críticas evoluciona a endoteliopatía y a coagulopatía con compromiso multiorgánico, que puede causar la muerte. El paciente con COVID-19 cursa con un cuadro similar a la influenza. Para mejorar la probabilidad del diagnóstico, es fundamental indagar por el contacto de contagio con pacientes con COVID-19 y el nexo epidemiológico o procedencia de países y ciudades con casos positivos. El SARS-nCoV-2 ha presentado una tasa de reproductividad básica (R0) de 2,68, un período medio de incubación de 5 a 7 días y del $40 \%$ al $50 \%$ de los casos son atribuibles a contacto con personas sin síntomas manifiestos o con síntomas leves ${ }^{(1-3)}$.

La glicoproteína Spike $S$ requerida para la entrada del virión a las células tiene como diana los receptores de la enzima convertidora de la angiotensina 2 (ECA-2), que se encuentran expresados en los neumocitos tipo II ${ }^{(2)}$. El proceso de activación de macrófagos y el síndrome de liberación de citocinas se plantean como patogénesis del SDRA y la linfohistiocitosis hemofagocítica vista en los casos graves como patogenia de la COVID-19. La activación del sistema inmunitario con citocinas de potente efecto proinflamatorio está implicada en la fisiopatología de la hipotensión y la disfunción pulmonar en el SDRA ${ }^{(2,3)}$.

\section{El cortisol en los procesos de inflamación}

Los pacientes críticamente enfermos están enfrentados a situaciones que modifican la respuesta de la glándula suprarrenal. En estos casos, existen condiciones asociadas a la situación crítica, que pueden influir negativamente en las acciones de cortisol desde el punto de vista clínico ${ }^{(4)}$. La secreción del cortisol es controlada por las neuronas del núcleo paraventricular del hipotálamo, que sintetizan y secretan la hormona liberadora de la corticotropina (CRH) al sistema portal hipofisario. La CRH, a su vez, estimula a las células corticotropas de la hipófisis anterior para la síntesis y liberación de la hormona adrenocorticotrópica (ACTH), que viaja por vía hematógena 
haciendo el blanco en las células de la corteza suprarrenal, para sintetizar y liberar los glucocorticoides a la circulación sistémica ${ }^{(5)}$. El cortisol regula su propia producción a través de un mecanismo de retroalimentación negativo. La corteza suprarrenal produce glucocorticoides, mineralocorticoides y andrógenos. Las múltiples acciones de los glucocorticoides involucran vías metabólicas, el transporte de iones, el mantenimiento del tono y la permeabilidad vascular, y a nivel del sistema nervioso central ejerce efectos paracrinos e intracrinos en el sistema inmunitario. El cortisol es transportado por la transcortina, su globulina transportadora, y el 15\% se une a la albúmina y solo una pequeña cantidad se encuentra no unida a las proteínas y viaja como cortisol libre biodisponible ${ }^{(6)}$.

La mayoría de las acciones del cortisol dependen de su unión al receptor de los glucocorticoides (RG) y de la translocación al interior de la célula del binomio cortisol/receptor de glucocorticoides. Las acciones metabólicas dependen de un mecanismo de transactivación y los efectos inmunológicos dependen de un mecanismo de transrepresión ${ }^{(4)}$. El RG contiene cuatro dominios: uno terminal $\mathrm{N}$ (dominio de transactivación), un dominio de unión central al ADN (ABD), un dominio de unión al ligando $C$ terminal (LBD) y un dominio bisagra, que conecta el ABD con el LBD ${ }^{(7)}$. Este receptor posee dos isoformas, $\mathrm{RG} \alpha$, que constituye el $90 \%$, y el RG $\beta$, que es estimulado durante la respuesta inflamatoria por las citocinas y produce una menor respuesta a los glucocorticoides como mecanismo de resistencia.

Un paciente críticamente enfermo es influenciado por factores que convergen en la activación del eje hipotálamo-hipofisario-suprarrenal (HHS), que incrementa la síntesis y la secreción de cortisol. El uso de esteroides inhalados o sistémicos puede disminuir su secreción durante el estrés agudo ${ }^{(8)}$. En la fase de estrés, se disminuye la síntesis de proteínas transportadoras de cortisol y, como consecuencia, aumenta su fracción libre ${ }^{(9)}$. Por otra parte, también se ha descrito resistencia de los RG por diferentes mecanismos ${ }^{(4)}$.

El término de actividad inadecuada de los corticosteroides fue acuñado en 2009 por Marik y describe elevados niveles séricos de cortisol y resistencia celular del RG ${ }^{(10)}$. El uso de esteroides en estrés agudo debe ser individualizado y se prefiere la medición del cortisol libre, sin omitir la posibilidad de resistencia en los RG.

\section{Esteroides y riesgo de infección}

El riesgo de infección aumenta con la dosis y la duración del tratamiento con corticosteroides y tiende a permanecer menor con dosis bajas, así sean acumuladas ${ }^{(11)}$. Sin embargo, se debe tener en cuenta la potencia del esteroide usado, porque a mayor potencia, es más probable que aumente el riesgo de infecciones. Su uso prolongado por más de 3 semanas tiene un efecto supresor de la inmunidad celular mediada por linfo- citos $\mathrm{T}$, que favorece la presencia de infecciones oportunistas (12). Las hospitalizaciones por infecciones fueron 2,5 veces más frecuentes en pacientes con consumo de esteroides, tabaquismo y FR positivo ${ }^{(13)}$. Las infecciones por hongos se han descrito más en los pacientes con Cushing endógeno.

Para los pacientes que consumen glucocorticoides, se recomienda la vacunación contra la influenza, aunque se sabe que los esteroides disminuyen su efecto protector. En este grupo de pacientes, las vacunas contra la varicela, la polio, el sarampión y el BCG están contraindicadas ${ }^{(14)}$.

\section{Uso de corticoides en coronavirus}

En las epidemias de 2002-2003 causadas por el SARS-CoV causante del síndrome respiratorio del Medio Oriente (MERS$\mathrm{CoV}$ ), la Organización Mundial de la Salud (OMS) no indicó prescripción de glucocorticoides en ningún tipo de dosis por el aumento de casos de ventilación invasiva y mortalidad relacionados con su uso ${ }^{(15)}$.

Es común usar corticosteroides en pacientes críticos, en quienes existen factores de confusión, ya que las infecciones aumentan los requerimientos de fluidos por la presencia de fiebre y taquipnea en situaciones donde los enfermos no pueden ingerir líquidos por la alteración de la consciencia; sin embargo, los estudios no han suministrado datos que definan las recomendaciones y solo muestran evidencia del posible daño y los efectos adversos de usar corticosteroides en el manejo de la COVID-19. Muchos estudios en COVID-19 son observacionales y poseen limitaciones metodológicas, aunque dicha evidencia clínica inconclusa no debería ser la razón para abandonar la opción del uso de corticosteroides en algunos pacientes con COVID-19 que posean una indicación clara.

Así como Rusell y colaboradores argumentan que los corticosteroides no deberían usarse en lesión pulmonar o en choque inducido por el nuevo coronavirus COVID-19, existen otros datos que soportan el uso de dosis bajas y moderadas por el hecho que en 41 pacientes críticamente enfermos sin infecciones secundarias u otras complicaciones se redujo la mortalidad y el tiempo de estancia en el hospital. La lesión pulmonar aguda (LPA) y el SDRA son parcialmente causados por la respuesta inmunitaria del huésped. Los corticosteroides suprimen la inflamación pulmonar, pero también inhiben la respuesta inmunitaria y el aclaramiento del patógeno ARN viral, elementos que deben tenerse en cuenta, además de los epidemiológicos, donde los estudios muestran alto riesgo de error debido a los múltiples factores de confusión ${ }^{(16-18)}$.

En Colombia, la Asociación Colombiana de Infectología (ACIN) dedicó un párrafo de sus recomendaciones para la actual pandemia, anotando que los corticosteroides no han demostrado utilidad en las epidemias previas de SARS y MERS, y en cambio pueden aumentar la eliminación de partículas virales y otros efectos secundarios propios de su grupo farmaco- 
lógico. Entre los muertos e infectados por SARS-COVID-19 que presentaron SDRA y recibieron metilprednisolona, fallecieron el $46 \%$ versus el $61 \%$ en el grupo comparador, con una reducción de riesgo HR de 0,38 (IC: 0,2-0,72) ${ }^{(19)}$.

Los efectos adversos relacionados con el tratamiento con glucocorticoides dependen de la dosis y de su potencia antiinflamatoria (Tabla 1). Los corticosteroides pueden causar síntomas de hipercortisolismo, especialmente en aquellos pacientes con mayor susceptibilidad, o hipoadrenalismo después de suspender su administración por supresión del eje HHS. Al administrarse en pacientes tratados con fármacos antirretrovirales, como el ritonavir, que actúa como un potente inhibidor de la citocromo P4503A, se incrementa la vida media del corticosteroide, lo que produce una supresión prolongada en la línea del tiempo del eje HHS. Debe también anotarse que la exposición a los disruptores endocrinos que modifican la actividad esteroidogénica puede, en el futuro, alterar la pronta recuperación de la función suprarrenal ${ }^{(20)}$.

Tabla 1. Equivalencia entre las dosis de diferentes preparados de esteroides

\begin{tabular}{l|c|c|c|c}
\multicolumn{1}{c|}{ Fármaco } & $\begin{array}{c}\text { DE en } \\
\text { mg }\end{array}$ & $\begin{array}{c}\text { Potencia } \\
\text { GC }\end{array}$ & $\begin{array}{c}\text { Potencia } \\
\text { MC }\end{array}$ & $\begin{array}{c}\text { Vida } \\
\text { media } \\
\text { (horas) }\end{array}$ \\
\hline Hidrocortisona & 20 & 1,0 & 1,0 & $8-12$ \\
\hline Prednisona & 5 & 4 & 0,8 & $18-36$ \\
\hline Metilprednisolona & 4 & 5 & 0,5 & $18-36$ \\
\hline Triamcinolona & 4 & 5 & 0 & $18-36$ \\
\hline Fludrocortisona & 2 & 10 & 125 & $18-36$ \\
\hline Deflazacort & 7,5 & 4 & 0,5 & $18-36$ \\
\hline Dexametasona & 0,75 & 25 & 0 & $36-54$ \\
\hline Betametasona & 0,5 & 30 & 0 & $36-54$ \\
\hline
\end{tabular}

DE: dosis equivalente; GC: glucocorticoide; MC: mineralocorticoide.

En pacientes que necesitaron esteroides, su retiro puede producir una crisis suprarrenal que amenace la vida. Debido a su vida media corta, la hidrocortisona se asocia, generalmente, a una recuperación más rápida de la función del eje HHS. El diagnóstico se realiza con la sospecha clínica y la medición de cortisol, sin el influjo del medicamento por tres vidas medias del fármaco utilizado; un cortisol sérico de la mañana menor de $3 \mu \mathrm{g} / \mathrm{dL}$ o un pico de cortisol sérico menor de $20 \mu \mathrm{g} / \mathrm{dL}$ posterior al estímulo con ACTH; generalmente, el cuadro se acompaña de hiponatremia. Debe anotarse que existe una predisposición individual por la que las personas susceptibles pueden tener supresión del eje HHS con ciclos cortos de esteroides de
5 días, incluso en dosis bajas. Estudios recientes mostraron insuficiencia suprarrenal en pacientes que tomaban prednisolona en $5 \mathrm{mg} / \mathrm{d}$ y una mortalidad aumentada después de la interrupción del tratamiento ${ }^{(21)}$.

El uso de corticosteroides en el paciente con COVID-19 quedará a criterio del médico y supeditado a otra indicación: choque séptico refractario, exacerbación de EPOC, crisis asmática o insuficiencia suprarrenal. Según el consenso de expertos de la Sociedad Torácica China, estos son los principios básicos para usar en el tratamiento con corticosteroides:

- Sopesar el riesgo-beneficio antes de su inicio.

- Uso prudente en pacientes críticamente enfermos con neumonía por COVID-19.

- Uso con precaución en pacientes con hipoxemia por enfermedad subyacente o en quienes los reciben crónicamente por su enfermedad de base.

- Al indicarlos, se recomienda administrar dosis bajas a moderadas $(\leq 0,5-1 \mathrm{mg} / \mathrm{kg} / \mathrm{d}$ de metilprednisolona o su equivalente) durante un ciclo corto menor o igual a 7 días ${ }^{(17)}$.

En los casos de sospecha de COVID-19 en pacientes con insuficiencia suprarrenal de base, debe realizarse una oportuna y rápida modificación al alta en las dosis de reemplazo con corticosteroides, como la propuesta para los "días enfermo" indicada en cualquier otra situación de incremento del estrés fisiológico. El doblar las dosis de reemplazo evita la crisis suprarrenal; la recomendación es mantener esta cantidad hasta que desaparezca la fiebre, lo que obliga al paciente a tener un adecuado suministro de medicamento en su casa para guardar el confinamiento en caso de que no requiera hospitalización ${ }^{(22,23)}$. Aquellos que padecen insuficiencia suprarrenal primaria o secundaria y usan hidrocortisona deben incrementar la dosis a $20 \mathrm{mg}$, cada 6 horas, y aquellos que reciben 10 a $15 \mathrm{mg} / \mathrm{d}$ de prednisolona deben tomar $10 \mathrm{mg}$ del mismo compuesto, cada 12 horas; si consumen dosis mayores, deben mantener la dosis o tomar al menos $10 \mathrm{mg}$ cada 12 horas. Si el paciente consume fludrocortisona, debe continuar con la dosis usual. En caso de crisis suprarrenal o presencia de vómito intenso y diarrea, se debe administrar inmediatamente 100 mg de hidrocortisona intramuscular como aplicación de emergencia y contactar al centro médico ${ }^{(14)}$. En general, se recomienda que estas dosis sean espaciadas y distribuidas uniformemente en el día y la noche. Es importante que los pacientes con insuficiencia suprarrenal mantengan las recomendaciones generales, las pautas de higiene, el lavado de manos, la limpieza de superficies, el cubrirse al toser, el uso de tapabocas y haber recibido las vacunas contra los gérmenes encapsulados.

Debe recordarse que el 5\% de la población consume corticosteroides para enfermedades inflamatorias o autoinmunitarias y que el uso de altas dosis de estos fármacos no requiere dosis adicionales en estos pacientes; su riesgo de morir depende de sus comorbilidades, el efecto inmunosupresor de los fármacos que utiliza y el trastorno inmunitario subyacente ${ }^{(24)}$. 
No existe evidencia actual que indique que padecer de insuficiencia suprarrenal aumente el riesgo de infectarse por SARS-nCoV-2 y presentar COVID-19; sin embargo, los pacientes que presentan enfermedad de Addison tienen un leve incremento en el riesgo de contraer infecciones debido a la alteración en su inmunidad natural y a los defectos en la actividad de los neutrófilos y las células natural killer ${ }^{(25)}$. Es también conocido que las infecciones respiratorias aumentan la mortalidad observada en pacientes con insuficiencia adrenal.

\section{Conclusiones}

La pandemia por COVID-19 trajo consigo muchos interrogantes sobre el efecto del proceso infeccioso en órganos y sistemas corporales, que ha obligado a los médicos y científicos de todo el mundo a emitir indicaciones para su tratamiento.
Muchas de estas no tienen aún estudios epidemiológicos robustos para respaldar estas recomendaciones, por lo que ahora, más que nunca, debemos alimentar nuestro conocimiento y utilizar el bien ponderado juicio clínico.

\section{Conflictos de interés}

Ninguno.

\section{Financiación}

Los autores no recibieron financiación para la realización de este documento.

\section{Agradecimientos}

Agradecemos a nuestros pacientes, que nos motivan cada día a seguir trabajando.

\section{Referencias}

1. Chen N, Zhou M, Dong X, Qu J, Gong F, Han Y, et al. Epidemiological and clinical characteristics of 99 cases of 2019 novel coronavirus pneumonia in Wuhan, China: a descriptive study. The Lancet. 2020;395(10223):507-13. doi: https://doi.org/10.1016/S0140-6736(20)30211-7.

2. Dhama K, Sharun K, Tiwari R, Sircar S, Bhat S, Malik YS, et al. Coronavirus Disease 2019 - COVID-19. Preprints. 2020;2020030001. doi: 10.20944/ preprints202003.0001.v1.

3. Huang C, Wang Y, Li X, Ren L, Zhao J, Hu Y, et al. Clinical features of patients infected with 2019 novel coronavirus in Wuhan, China. The Lancet. 2020;395(10223):497-506. DOI:https://doi.org/10.1016/S01406736(20)30183-5.

4. Guerrero J. Para entender la acción del cortisol en inflamación aguda: una mirada desde la glándula suprarrenal hasta la célula blanco. Rev Med Chile. 2017;145(2):230-9. doi: http://dx.doi.org/10.4067/S003498872017000200011.

5. Casanueva F, Ghigo E. Hypothalamic Pituitary Diseases. Springer Nature; 2018. doi: https://doi.org/10.1007/978-3-319-44444-4.

6. Melmed S. The Pituitary. 3. ${ }^{a}$ edición. Academic Press; 2010.

7. Slominski RM, Tuckey RC, Manna PR, Jetten AM, Postlethwaite A, Raman C, et al. Extra-adrenal glucocorticoid biosynthesis: implications for autoimmune and inflammatory disorders. Genes Immun. 2020. doi. 10.1038/ s41435-020-0096-6.

8. Ahmet A, Kim H, Spier S. Adrenal suppression: A practical guide to screening and management of this under-recognized complication of inhaled corticosteroid therapy. Allergy Asthma Clin Inmunol. 2011;7(1):13. doi: 10.1186/1710-1492-7-13.

9. Venkatesh B, Imeson L, Kruger P, Cohen J, Jones M, Bellomo R, et al. Elevate plasma - free cortisol concentrations and ratios are associated with increased mortality even in the presence of statin therapy in patients with severe sepsis. Crit Care Med. 2015;43(3):630-5. doi: 10.1097/ CCM.0000000000000750.

10. Marik PE. Critical illness-related corticosteroid insufficiency. Chest. 2009;135(1):181-93. doi: 10.1378/chest.08-1149.

11. Chen HS, Tsai WP, Leu HS, Ho H, Liou L. Invasive fungal infection in systemic lupus erythematosus: an analysis of 15 cases and a literature review. Rheumatology (Oxford). 2007;46(3):539-44. doi: 10.1093/rheumatology/ kel343.

12. Klein NC, Go Ch, Cunha BA. Infections associated with steroid use. Infect Dis Clin North Am. 2001;15(2):423-32, vii. doi: 10.1016/s08915520(05)70154-9.

13. Van Everdingen AA, Jacobs JW, Siewertsz DR, Bijlsma JW. Low-dose prednisone therapy for patients with early active rheumatoid arthritis: clinical efficacy, disease-modifying properties, and side effects: a randomized, dou- ble-blind, placebo-controlled clinical trial. Ann Intern Med. 2002;136(1):112. doi: 10.7326/0003-4819-136-1-200201010-00006.

14. Cutolo M, Seriolo B, Pizzorni C, Secchi ME, Soldano S, Paolino S, et al. Use of glucocorticoids and risk of infections. Autoimmun Rev. 2008;8(2):153-5. doi: 10.1016/j.autrev.2008.07.010.

15. World Health Organization. Clinical of Management of severe acute respiratory infection when novel coronavirus (2019-nCoV) infection is suspected: interim guidance [Internet]. Disponible en: https://apps.who.int/iris/ handle/10665/330893.

16. Rusell C, Millar JE, Baillie K. Clinical evidence does not support corticosteroid treatment for 2019-nCoV lung injury. The lancet. 2020;395(10223):473-5. doi: http://doi.org/10/1016/S0140-6736(20)30317-2.

17. Shang L, Zhao J, Hu Y, Du R, Cao B. On the use of corticosteroids for 2019 nCoV pneumonia. Lancet. 2020;395(10225):683-8. doi: https://doi. org/10.1016/S0140-6736(20)30361-5.

18. Chen RC, Tang XP, Tan SY, Liang BL, Wan ZY, Fang JQ et al. Treatment of severe acute respiratory syndrome with glucosteroids: the Guangzhou experience. Chest. 2006;129(6):1414-52. doi: 10.1378/chest.129.6.1441.

19. Saavedra Trujillo $\mathrm{CH}$. Consenso colombiano de atención, diagnóstico y manejo de la infección por SARS-COV-2/COVID-19 en establecimientos de atención de la salud. Recomendaciones basadas en consenso de expertos e informadas en la evidencia. Infectio. 2020;24(3):1-153. doi: http://dx.doi. org/10.22354/in.v24i3.851.

20. Scaroni C, Armigliato M, Cannavo S. COVID-19 outbreak and steroids administration: are patients treated for Sars-Cov-2 at risk of adrenal insufficiency? J Endocrinol Invest. 2020;1-2. doi: 10.1007/s40618-020-01253-1.

21. Mebrahtu TF, Morgan AW, Kelley A, Baxter PD, Stewart PM, Pujades-Rodriguez M. Dose dependency of iatrogenic glucocorticoid excess and adrenal insufficiency and mortality: a cohort study in England. J Clin Endocrinol Metab. 2019;104(9):3757-3767. doi: 10.1210/jc.2019-00153.

22. Puig-Domingo M, Marazuela M, Giustina A. COVID-19 and endocrine disease: A statement from the European Society of Endocrinology. Endocrine. 2020;69(1):2-5. doi: 10.1007/s12020-020-02294-5.

23. Alextlc.org. COVID-19 :New UK steroid guidance issued. Advice for patients who take replacement steroids (hydrocortisone, prednisolone, dexamethasone or plenadren) for pituitary/adrenal insufficiency [Internet]. Disponible en: https://www.alextlc.org/covid-19-new-uk-steroid-guidance-issued/.

24. Kaiser U, Mirmira RG, Stewart P. Our response to COVID-19 as Endocrinologist and Diabetologist. J Clin Endocrinol Metab. 2020;105(5):dgaa148. doi: 10.1210/clinem/dgaa148.

25. Bancos I, Hazeldine J, Chortis V, Hampson P, Taylor AE, Lord JM, et al Primary adrenal insufficiency is associated with impaired natural killer cell function: a potential link to increased mortality. Eur J Endocrinol. 2017;176(4):471-80. doi: 10.1530/EJE-16-0969. 